\title{
Contralateral hemiparesis following herpes zoster ophthalmicus
}

\author{
CHRISTINE GASPERETTI, SUN KYU SONG \\ From the Division of Neuropathology, Mount Sinai School of Medicine, The City University of New York, \\ USA
}

SUMMARY A case of herpes zoster ophthalmicus was followed, after a latent period of four weeks, by contralateral hemiparesis. An attempt is made to clarify the anatomical relationships involved in the pathogenesis of postherpetic cerebral complications. Detailed presentation of ${ }^{\circ}$ neuropathological findings in this case may provide evidence of vasculitis in the syndrome, with better understanding of the pathological anatomy.

Anastasoupoulos in $1958^{1}$ described a syndrome of contralateral hemiparesis occurring subsequent to herpes zoster ophthalmicus and proposed that this syndrome might have resulted from the spread of inflammation from the trigeminal nerve ganglion to nearby small vessels. Twenty-five such cases have been reviewed. There has not, however, so far been a description of the specific process involved in the pathogenesis of this syndrome. We present a case of herpes zoster ophthalmicus followed by contralateral hemiparesis in which neuropathological findings provide evidence of focal vasculitis in the meningeal arteries as well as small blood vessels in the distribution of the middle and posterior cerebral arteries. These findings support the probable role of direct spread of the inflammation process in the pathogenesis of the syndrome.

\section{Case history}

A 79-year-old woman was admitted with a 1 week history of herpes zoster ophthalmicus on the right side. About four weeks after the onset of the herpetic symptoms, she developed a flaccid weakness of the left upper extremity that was preceded by mental confusion, lethargy, urinary incontinence and hypothermia for several days. Neurological examination revealed left hemiparesis that was more marked in the upper extremity and accompanied by a lower facial weakness. Deep tendon reflexes were absent in

Address for reprint requests: Dr SK Song, Division of Neuropathology, Mount Sinai School of Medicine, One Gustav L. Levy Place, New York, NY 10029, USA.

Received 21 October 1983 and in final revised form 12 August 1984.

Accepted 23 August 1984. the left arm but were exaggerated in the left leg. There were no pathological reflexes. She showed a mild organic mental syndrome. The cerebrospinal fluid contained 19 red blocd cells and 26 white blood cells per $\mathrm{mm}^{3}$ of which $13 \%$ were polymorphonuclear cells and $87 \%$ were lymphocytes. The fluid was clear and colorless and its culture did not grow any microorganism. Electroencephalogram showe "diffuse cerebral dysfunction with right sided accentuक tion". Isotope scan of the brain showed normal uptake patterns. Her neurological condition did not improve and she died thirty-eight days after the onset of the herpetis symptom.

\section{Postmortem findings}

Necropsy was performed approximately 12 hours after death. It showed generalised atherosclerosis with mild left ventricular hypertrophy of the heart and acute passive congestion of lungs in the bilateral upper lobes. The liver and kidneys showed moderate to severe congestion.

The nervous system showed the following changes:

\section{Right Semilunar ganglion of the trigeminal nerve}

There were large numbers of lymphocytes infiltrating throughout the ganglion and the pre- and post-ganglionic portions of the trigeminal nerve. Ganglion cells were not affected.

\section{Right trigeminal nerve root}

The root exit at the right lateral aspect of the mid pons showed patchy areas of demyelination with a loss of axons that were replaced by small numbers of macrophages. The changes could be traced caudally to involve the spinal tract of the trigeminal nerve nucleus in the pons and the rostral medulla on the same side. There were no abnormalities in the left side.

3 Thalamus and corpus striatum

Several well circumscribed recent infarcts were encountered in the right corpus striatum, bilateral thalamus and 
the right hippocampal formation of the temporal lobe. These areas were destroyed by liquefaction necrosis and were replaced by compact aggregates of macrophages. Anatomical distribution suggested the involvement of the right middle and posterior cerebral arteries.

\section{Leptomeninges and meningeal arteries:}

Many of the small to medium sized meningeal arteries near the uncus and the hippocampal formation of the right temporal lobe were surrounded and invaded by large numbers of lymphocytes and histiocytes with fibrinoid necrosis of the tunica media of the vessel walls. The intima was oedematous and the lumens were frequently obliterated by well organised thromboses. (figs 1,2 ) In addition, the leptomeninges in this vicinity were infiltrated by similar inflammatory cells. (fig 3) Major portions of the arteries forming the Circle of Willis showed no inflammatory changes. The leptomeninges in other areas of the central nervous system showed no abnormalities.

\section{Immunoperoxidase reaction:}

Paraffin sections of the formalin-fixed tissue samples taken from the right semilunar ganglion, right hippocampal for-

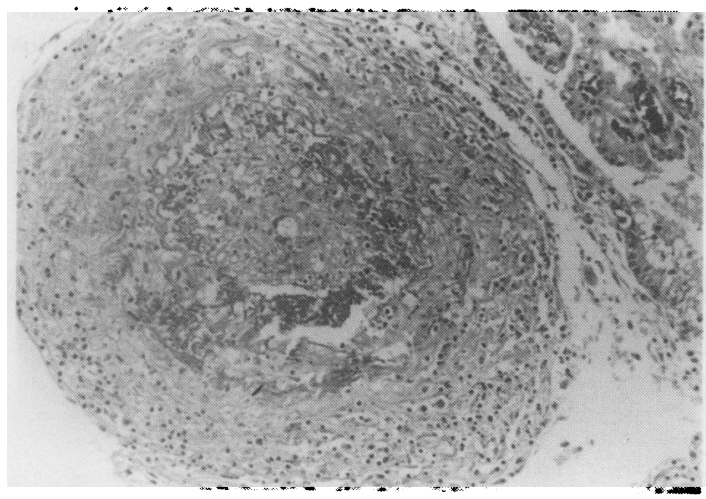

Fig 1 A thrombosed meningeal artery with arteritis near right hippocampal formation. Ependymal lining and choroid plexus are at right upper corner. $(\times 340)$

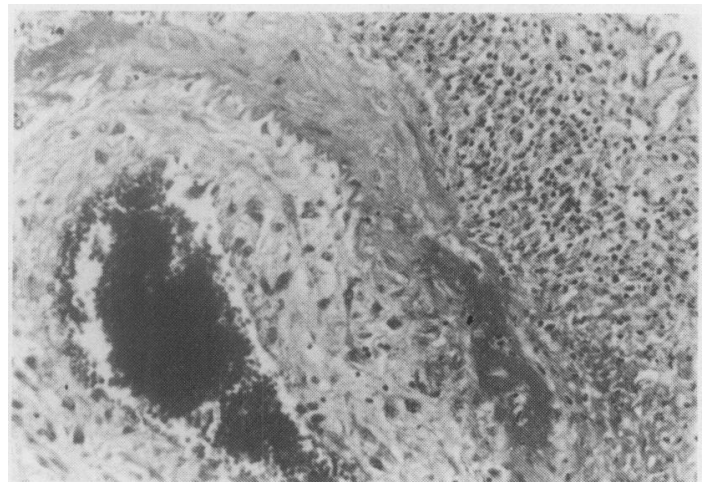

Fig 2 Arteritis showing inflammatory infiltration, fibrinoid necrosis of tunica media with destruction of elastic lamina and oedematous thickening of intima. $(\times 500)$

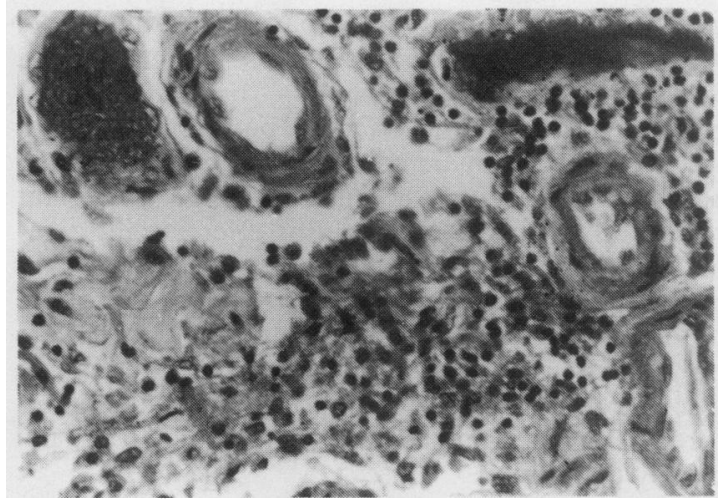

Fig 3 Lymphocytic infiltration of adjacent meninges. $(\times 1250)$

mation and the right thalamus did not show detectable evidence of Herpes antigen activity.

\section{Discussion}

The occurrence of contralateral hemiparesis subsequent to herpes zoster ophthalmicus, a syndrome which affects both men and women (ratio of 2:1) with average age of $55 \mathrm{yr}$ (5-96 in reported cases), has been well recognised. Onset is gradual, and is heralded by pain in the eye with vesicular mucocutaneous lesions on the forehead, nose and cheek on the same side. Patients may develop various ophthalmic signs that include visual blurring, diplopia, ptosis and amaurosis. After a variable period of 3 to 5 weeks (up to 23 weeks have been reported), there is onset of contralateral hemiparesis. In three cases, aphasia was noted in association with hemiparesis, as well as contralateral hemianopia in two other cases. In nearly half of the reported cases, changes in mental status: memory impairment, confusion, disorientation, psychosis were noted.

Onset of hemiparesis is usually acute though in two cases it was reported to be slow and progressive. For the most part the course of illness is relatively benign with gradual improvement. In many instances, steroid or anticoagulant therapy was recommended. Some patients appear to progress to more extensive involvement of the nervous system.

A number of hypothesis have been postulated to explain the occurrence of contralateral hemiparesis following herpes zoster ophthalmicus. Anastsasoupoulos ${ }^{1}$ in 1958 considered that the ipsilateral disturbance was caused by spread of an inflammatory process from the affected ganglion to nearby blood vessels causing thrombosis and distal embolisation. MacKenzie ${ }^{2}$ has suggested that compromised immune function could account for persistent 
infection in this subgroup of patients. Others have suggested that the hemiparesis is preceded by a more diffuse viral encephalitis ${ }^{3}$ or by a diffuse granulomatous angiitis. ${ }^{4}$ One report regarded that the two events may be connected by chance..$^{5}$ Many authors interpreted radiological findings of vascular obliterations as due to vasculitis affecting intracranial arteries. ${ }^{46-8}$ (Table 1) Only two reports contained postmortem findings confirming the presence of vasculitis. ${ }^{12}$ (Table 2) Unfortunately, they were only included as one of the case examples in the discussion of "granulomatous angiitis" and therefore the specific anatomical relationships were not clearly defined.

In our case, similarly, neuropathological findings are consistent with focal vasculitis in the meningeal arteries. In particular, this is the first described case in which anatomical relationship between the herpetic lesion and the subsequent development of vasculitis is clarified. Vecht and de Sande ${ }^{13}$ emphasised the importance of the necessary step as support of direct spread of the inflammatory process from the ganglion to nearby small vessels. The focal nature of the lesion and the involvement of predominantly small vessels may explain the varying time interval seen between two clinical events as well as the absence of seizures, fever, and leukocytosis that are characteristic of a more diffuse encephalitic process.

MacKenzie $^{2}$ interpreted the pattern of vascular involvement seen in four patients with the syndrome as suggestive of spread along the intracranial branches of the ophthalmic nerve followed by involvement of the cerebral arteries and more generalised spread via the subarachnoid space. However, none of these cases had histopathological confirmation.

The findings in the case presented here indicate the localised nature of the inflammatory changes in the meninges and meningeal arteries that are situated in the immediate vicinity of the affected semilunar ganglion. Although the exact pathogenesis is not clearly understood, the patterns of the inflammatory reactions appear difficult from that of "granulomatous angiitis".

Doyle et al. ${ }^{4}$ reported that a herpes-like virus was identified in smooth muscle cells of the middle cerebral artery in a patient who developed post-herpetic contralateral hemiparesis which ultimately progressed to massive cerebral infarction and death. Linnemann ${ }^{15}$ and Reyes ${ }^{16}$ described virus-like particles in two separate cases of granulomatous angiitis. McComas and Gutman ${ }^{17}$ described a case in which temporal lobe and meningeal biopsy specimens were examined by electronmicroscopy and various vir

Table 1 Clinical laboratory findings

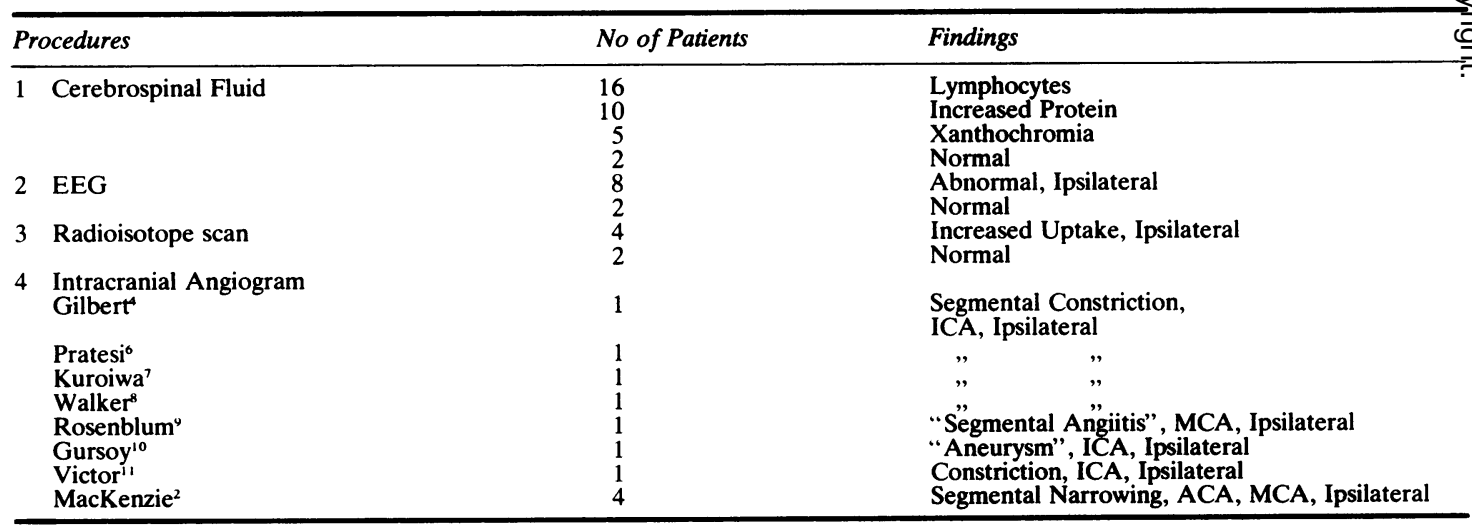

Abbreviations: ICA $=$ Internal carotid artery; $\mathrm{MCA}=$ Middle cerebral artery; ACA $=$ Anterior cerebral artery.

Table 2 Postmortem findings

\begin{tabular}{lll}
\hline Authors & Anatomical localisation & Pathological description \\
\hline Rosenblum $^{\prime}$ & Basal Ganglia, Int Capsule & Infarct \\
& Trigeminal Nerve & Demyelination, Lymphocytes, Reticulum Cell Sarcoma \\
& Meningeal Arteries, Frontal Lobe & Mononuclear Cells, Fibrinoid Necrosis \\
Kolodny'2 & Basal Ganglia, Diencephalon, Temporal Lobe & Infarct \\
& Spinal Cord, Lateral Columns & Degeneration \\
& Trigeminal Nerve, Semilunar Ganglion, & Inflammatory infiltrates \\
& Oculomotor Nerve & Inflammation, Fibrinoid Necrosis \\
\hline
\end{tabular}


ological methods that failed to uncover the evidence of specific viral infection.

Based on evidence of a vasculitis process in this syndrome, one must consider anticoagulant or steroid medications in the treatment of these patients. No clinical trials have been reported to evaluate the rationale and efficacy of these or other therapeutic agents in halting the progression of inflammation or in identifying those aspects of the syndrome which have prognostic validity. Landi ${ }^{18}$ has suggested that clinicians avoid stress on the vascular system which might lead to further compromise of blood supply.

During the last decade, systemic antiviral chemotherapeutic agents, such as vidarabine and acyclovir, have shown promising results in the treatment of varicella zoster. ${ }^{19-23}$ But their effectiveness is mostly dependent upon early administration of the agent following the initial onset of viral infection. It is not known, however, whether the administration of early chemotherapy would reduce the occurrence of post-herpetic inflammatory complications. Based on morphological similarities of arteritis between this case and the general histopathological profiles of polyarteritis nodosa and temporal arteritis, it may be assumed that the role of antiinflammatory agents may be greater in the management of this syndrome, once the signs of this syndrome have manifested.

In conclusion, pathological anatomy of the postherpetic hemiparesis syndrome described here clarifies the localised nature of inflammatory changes in the immediate vicinity of the affected semilunar ganglion. The changes extend to involve leptomeninges and meningeal blood vessels over the hippocampal formation apposing the medial portion of the middle cranial fossa. The outstanding feature of the inflammatory changes is arteritis with fibrinoid necrosis of the tunica media affecting many of the small to medium sized arteries leading to occlusion of their lumens by thrombosis. The arteritis and subsequent thrombosis result in multiple focal infarction in the temporal lobes, occipital lobe, basal ganglia and thalamus which correspond to the distribution of the middle and posterior cerebral arteries on the same side. It is not clearly understood, however, whether the meningeal arteritis is a part of the original infection or a post-infectious immunological reaction.

\section{References}

' Anastasopoulos F, Routsomas K, Ierodiakonou CS. Ophthalmic herpes zoster with contralateral hemiplegia.J Neurol Neurosurg Psychiatry 1958;21:210-2.

${ }^{2}$ MacKenzie RA, Forbes GS, Karnes WE. Angiographic findings in herpes zoster arteritis. Ann Neurol 1981; 10:458-64.

${ }^{3}$ Dolin R, Reichman RC, Mazur MH, Whitley RJ. Herpes zoster-varicella infections in immunosuppressed patients. Ann Int Med 1978;89:375-88.

${ }^{4}$ Gilbert GJ. Herpes zoster ophthalmicus and delayed contralateral hemiparesis. JAMA 1974;299:302-4.

${ }^{5}$ Barontini F, Tonini R. Contoversa complicanza dell herpes zoster oftalmico: l'emiparesi alterna tardiva. Riv Nerv Ment 1979; 100:275-87.

- Pratesi R, Freemon FR, Lowry JL. Herpes zoster ophthalmicus with contralateral hemiplegia. Arch Neurol 1977;34:640-1.

${ }^{7}$ Kuroiwa Y, Furukawa T. Hemispheric Infarct after Herpes zoster ophthalmicus: CT and angiography. Neurology (NY) 1981;31:1030-2.

${ }^{8}$ Walker RJ, Gammal TE, Allen MB. Cranial arteritis associated with herpes zoster: case report with angiographic findings. Radiology 1973;107:109-10.

${ }^{4}$ Rosenblum WI, Hadfield MG. Granulomatous angiitis of the nervous system in cases of herpes zoster and lymphosarcoma. Neurology (Minneap) 1972;22: 348-54.

${ }^{10}$ Gursoy G, Atkin E, Bahar S, Tolun R, Ozden B. Postherpetic aneurysm in intrapetrosal portion of the internal carotid artery. Neuroradiology 1980; 19: $279-82$.

"Victor DI, Green WR. Temporal artery biopsy in herpes zoster ophthalmicus with delayed arteritis. Am J Ophthalmol 1976;82 4:628-30.

12 Kolodny EH, Rebeiz JJ, Caviness VS, Richardson EP. Granulomatous angiitis of the central nervous system. Arch Neurol 1968;19:510-24.

${ }^{13}$ Vecht CJ, DeSande JJ. Hemispheric infarction after herpes zoster ophthalmicus. Neurology (NY) 1982;32:914.

14 Doyle PW, Gibson G, Dolman CL. Herpes zoster ophthalmicus with contralateral hemiplegia: identification of cause. Ann Neurol 1983;14:84-85.

is Linnemann CC, Alvira MM. Pathogenesis of varicellarzoster angiitis in the central nervous system. Arch Neurol 1980;37:239-40.

${ }^{16}$ Reyes MG, Fresco R, Chokrverty S, Salud EQ. Viruslike particles in granulomatous angiitis of the central nervous system. Neurology (Minneap) 1976;26:7979.

${ }^{17}$ McComas CF, Gutmann L. Reply to "Hemispheric infarction after herpes zoster ophthalmicus". Neurology (NY) 1982;32:914-5.

${ }^{18}$ Landi G, Calloni MV, Scalato G. Transient contralateral hemiplegia after ophthalmic zoster: therapeutic problems in elderly patients. $J$ Neurol 1981;224:297-300.

${ }^{19}$ Weller TH. Varicella and herpes zoster. $N$ Engl $J$ Med 1983;309: 1434-40.

${ }^{20}$ Whitley RJ, Ch'ien LT, Dolin R, Galasso GJ, Alford Jr, CA. Eds: Adenine Arabinoside Therapy of Herpes Zoster in the Immunosuppressed. NIAID Collaborative Antiviral Study. N Engl J Med 1976;294: 1193-9.

${ }^{21}$ Whitley RJ, Soong SJ, Dolin R. Early vidarabine therapy to control the complications of herpes zoster in immunosuppressed patients. $N$ Engl J Med 1982;307:971-5.

22 Bean B, Bean C, Balfour Jr, HH. Acyclovir Therapy for Acute Herpes Zoster. Lancet 1982;II: 118-21.

${ }^{23}$ Balfour Jr, HH, Bean B, Laskin OL, et al. Burrows Wellcome collaborative acyclovir study group: acyclovir halts progression on herpes zoster in immunocompromised patients. N Engl J Med 1983;308: 1448-53. 